CLINICAL STUDY

\title{
Risk of elevated resting heart rate on the development of type 2 diabetes in patients with clinically manifest vascular diseases
}

\author{
Remy H H Bemelmans ${ }^{1}$, Annemarie M J Wassink ${ }^{1}$, Yolanda van der Graaf ${ }^{2}$, Hendrik M Nathoe ${ }^{3}$, \\ Joris W P Vernooij ${ }^{1}$, Wilko Spiering ${ }^{1}$, Frank L J Visseren ${ }^{1}$ on behalf of the SMART Study Group ${ }^{\dagger}$ \\ ${ }^{1}$ Department of Vascular Medicine, ${ }^{2}$ Julius Center for Health Sciences and Primary Care and ${ }^{3}$ Department of Cardiology, University Medical Center Utrecht, \\ PO Box 85500, 3508 GA Utrecht, The Netherlands
}

(Correspondence should be addressed to F L J Visseren; Email: f.l.j.visseren@umcutrecht.nl)

${ }^{\dagger}$ Members of the SMART Study Group are listed in the acknowledgment section.

\begin{abstract}
Objective: Sympathetic nerve activation is causally related to insulin resistance as both a cause and a consequence. Resting heart rate (RHR) reflects sympathetic nerve activity. We investigated the effect of RHR on the incidence of type 2 diabetes mellitus (T2DM) in patients with clinically manifest vascular diseases.

Design: Data were used from the second manifestations of arterial disease (SMART) study: a prospective cohort study of patients with clinically manifest vascular diseases $(n=3646)$.

Methods: RHR was obtained using an electrocardiogram. Patients were followed up for incident type 2 diabetes $(n=289)$ during a median period of 5.5 (interquartile range 3.2-8.4) years. The relation between RHR and incident T2DM was estimated by Cox proportional hazard analysis. As age was an effect modifier $(P=0.048)$, analyses were stratified for age.

Results: Patients in quartile 4 (Q4) of RHR had a 65\% increased risk of T2DM compared with those in Q1 (reference; hazard ratios (HR), 1.65; 95\% confidence interval (95\% CI), 1.15-2.36) adjusted for age, gender, smoking, estimated glomerular filtration rate, systolic blood pressure, location of vascular disease, and antihypertensive medication. Every 10 beats per minute (bpm) increase in RHR increased the risk for T2DM with $10 \%$ (HR, 1.10; 95\% CI, 1.00-1.21) in the total population. This risk was particularly high in subjects aged 55-63 years (per 10 bpm: HR, 1.22; 95\% CI, 1.04-1.43) and was independent of the location of vascular disease and beta-blocker use.

Conclusions: Increased RHR, an indicator of sympathetic nerve activity, is associated with an increased risk for T2DM in patients with manifest vascular diseases, particularly in middle-aged patients.
\end{abstract}

European Journal of Endocrinology 166 717-725

\section{Introduction}

Insulin resistance and elevated sympathetic nerve activity are closely related, but the precise pathophysiological relation is complex and only partially understood. Visceral obesity is associated with a more pronounced sympathetic nerve activity than subcutaneous obesity (1), probably to a large extent caused by elevated serum levels of insulin and leptin (2). Administration of insulin in humans increases muscle sympathetic nerve activity, the reference standard of measuring sympathetic nerve activity (3), by a direct effect on insulin receptors in the $\mathrm{CN}(4,5)$. Plasma leptin levels are independently associated with resting heart rate (RHR) in healthy male subjects (6). Furthermore, the infusion of leptin in rats increases the heart rate, through leptin receptors in the $\mathrm{CN}(7,8)$.

The causal relation between sympathetic nerve activity and insulin resistance could also be the other way around. Acute reflex activation of the sympathetic nerve system is reported to induce acute insulin resistance in humans (9). Moreover, chronically increased sympathetic nerve activity can precede the development of insulin resistance and obesity $(10,11)$. RHR reflects sympathetic tone, and correlates with muscle sympathetic nerve activity and noradrenaline serum levels (12). An increased RHR identifies subjects at higher risk for developing cardiovascular disease and mortality $(13,14)$. Furthermore, an increased RHR at baseline is independently associated with an increased risk for developing type 2 diabetes mellitus (T2DM) in healthy populations $(15,16,17,18,19)$ and in subjects with obesity or impaired glucose tolerance (20) but this has not been shown in patients with vascular diseases.

Identifying subjects at high risk for developing T2DM is important, as diabetes is a strong risk factor for cardiovascular morbidity and mortality $(21,22)$ and patients with diabetes have a reduced quality of life (23). 
Furthermore, the risk for T2DM can be reduced through lifestyle interventions and medication (24). Patients with clinically manifest vascular diseases are at particularly high risk of developing new vascular events and T2DM given shared pathophysiological pathways ('common soil' hypothesis) of diabetes and atherosclerosis, such as insulin resistance and low-grade inflammation (25). As increased sympathetic nerve activity, reflected by an elevated RHR, is associated with increased risk for vascular diseases and mortality on the one hand $(13,26)$, and diabetes on the other hand $(15,16,17,18$, $19,20)$, increased sympathetic tone may also be part of the shared pathophysiology in the development of vascular diseases and T2DM. Patients with vascular diseases who have a particular high risk for the development of diabetes are an ideal group for preventive measures, given their high risk and the fact that they are already receiving medication and lifestyle advice, in contrast to the general population. Therefore, in this study we investigated the relation between RHR and incidence of T2DM during follow-up in a cohort of patients with clinically manifest vascular diseases.

\section{Subjects and methods}

\section{Study design and patients}

In this study, data were used from patients enrolled in the second manifestations of arterial disease (SMART) study, an ongoing single-center prospective cohort study carried out at the University Medical Center Utrecht. This study started in September 1996. Patients aged 18-80 years, referred to our institution with clinically manifest atherosclerotic vascular diseases, were included. Patients with terminal malignant disease, those not independent in daily activities (Rankin scale >3), and not sufficiently fluent in Dutch were not included.

The aims of the SMART study were to determine i) the risk factors for atherosclerosis, ii) prevalence of additional vascular disease, and iii) incidence of future cardiovascular events and type 2 diabetes. The study complied with the Declaration of Helsinki Principles; the Medical Ethics Committee of the UMC Utrecht, The Netherlands, approved the study and all patients gave written informed consent. Patients were asked to complete a health questionnaire covering medical history, risk factors, smoking habits, and medical treatment. A standardized diagnostic protocol consisting of physical examination and laboratory testing in a fasting state was followed. A more detailed description of the design of the study has been published previously (27).

For this study, data were used from patients $(n=5280)$ enrolled in the SMART cohort from September 1996 to March 2010 with a recent diagnosis or history of coronary artery disease (CAD), cerebrovascular disease (CVD), peripheral arterial disease (PAD), or abdominal aortic aneurysm (AAA).

Patients who had died $(n=480)$ or were lost to followup $(n=91)$ before the assessment of incident T2DM started in 2006 (see Follow-up and assessment of T2DM) were excluded (Fig. 1). Patients with diabetes mellitus (type 1 or 2 ) at study inclusion $(n=809)$, defined as a referral diagnosis of diabetes, self-reported diabetes (use of glucose-lowering agents), or a known history of diabetes, were excluded. To make sure that all patients with diabetes at baseline were excluded, subjects without a history of diabetes, but with the combination of a fasting plasma glucose level $\geq 7.0 \mathrm{mmol} / \mathrm{l}$ at baseline and receiving treatment with glucose-lowering agents within 1 year after baseline were considered as having diabetes at baseline and were excluded $(n=50)$. Furthermore, patients without a study electrocardiogram (ECG; $n=85$ ) or without sinus rhythm on ECG $(n=169)$ were excluded, leaving a total of 3646 patients for analyses (Fig. 1).

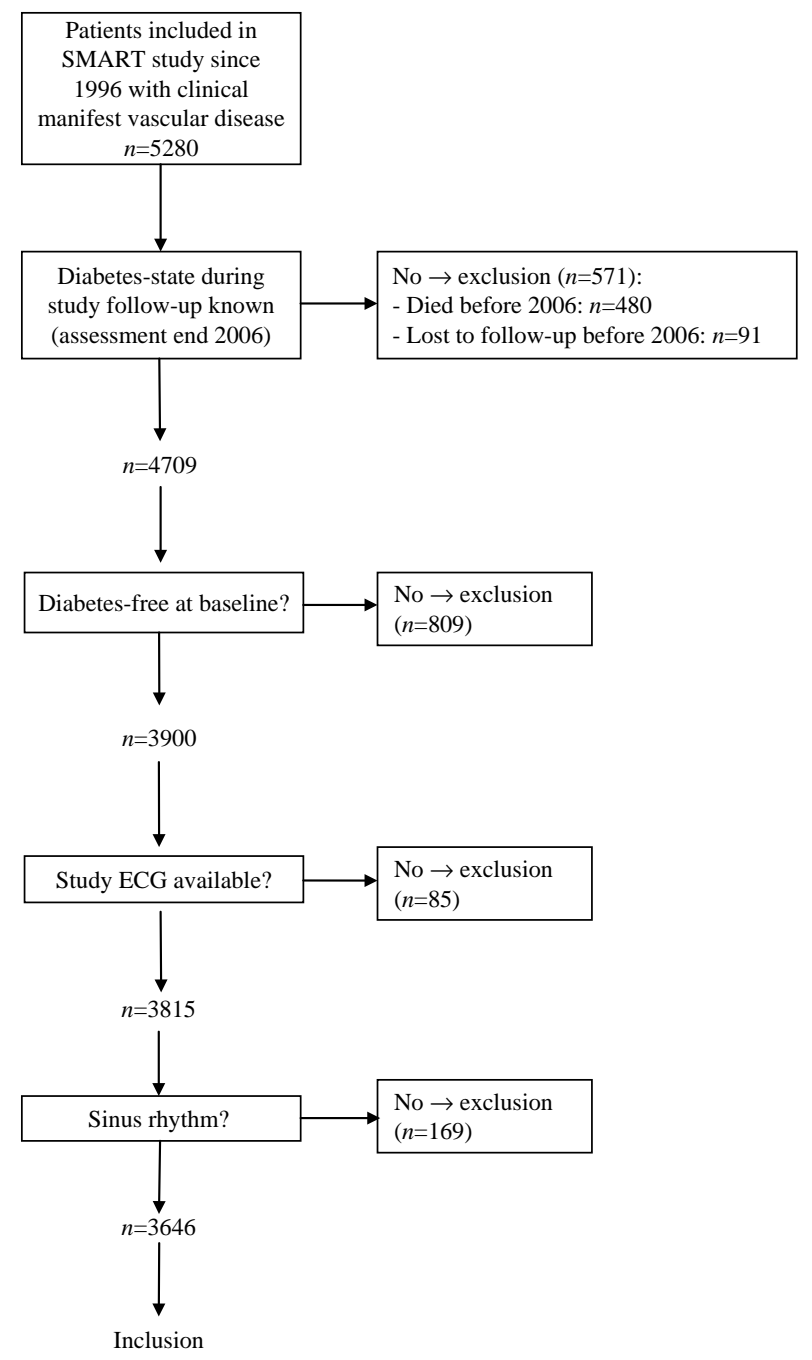

Figure 1 Flow chart. 


\section{Measurement of RHR}

At study inclusion, a 12-lead ECG was obtained after the patient had rested in supine position for $5 \mathrm{~min}$. RHR was calculated using the digitally stored 12-lead 10-s data, by dividing the number of $\mathrm{R}-\mathrm{R}$ intervals (number of QRS-complexes minus one) by the time difference between the first and last beat, and the result was converted to beats per minute (bpm). This calculation was performed using the Marquette 12SL analysis program (General Electric Healthcare, Hoevelaken, The Netherlands).

\section{Follow-up and assessment of type 2 diabetes}

The main outcome of interest for this study was incident T2DM. All participants that had been included until June 2006 without diabetes at baseline received a questionnaire in the period between June and December 2006 to assess the incidence of T2DM after study inclusion. After 2006, they were biannually asked to complete this questionnaire. Patients were asked whether they had diabetes and if 'yes', patients received a supplementary questionnaire regarding date of diagnosis, initial treatment (diet, oral medication, or insulin), current treatment, and family history of diabetes. If the answers were incomplete or unclear, patients and/or their general practitioner were called on the phone for further information. To validate the diagnosis of diabetes, two independent physicians audited and classified all diabetes cases. Furthermore, cross-validation with the hospital diagnosis registry revealed that none of the patients who reported not to have diabetes had a physician's diagnosis of diabetes.

Follow-up duration (years) was defined as the period between study inclusion and date of diagnosis of T2DM, date of death, date of loss to follow-up, or the preselected date of 1 March 2010. From 1996 until 1 March 2010, a total of $72(2 \%)$ patients were lost to follow-up.

\section{Data analyses}

Continuous variables are expressed as mean \pm s.D. when normally distributed or as median (interquartile range (IQR)) in case of skewed distribution. Categorical variables are expressed as numbers (percentage). Single imputation methods were used to reduce missing covariate data for smoking status $(n=15 ; 0.4 \%)$, body mass index (BMI; $n=5 ; 0.1 \%)$, systolic blood pressure (SBP; $n=5 ; 0.1 \%$ ), and estimated glomerular filtration rate (eGFR, calculated using the modification of diet in renal disease formula; $n=18 ; 0.5 \%$ ), as this method reduces the chance for bias compared with complete case analysis.

The incidence rate of T2DM was calculated as the number of new T2DM patients divided by total amount of person-years during the study follow-up. Corresponding 95\% confidence intervals (95\% CIs) were calculated using Fisher's exact test. The relation between RHR and incident type 2 diabetes was quantified with Cox proportional hazard analysis. Results are expressed as hazard ratios (HR) with 95\% CI. Patients were censored if they died or were lost to follow-up. The proportional hazard assumption was confirmed by testing the correlations between scaled Schoenfeld residuals for RHR and time. No significant nonproportionality $(P<0.05)$ was observed.

HR on incident T2DM were calculated per quartile RHR, using patients in quartile 1 (Q1) as reference category. Second, linear regression analyses were performed to estimate the risk of incident T2DM per 10 bpm increase in RHR, for the whole study population and after stratification for age in tertiles $(<55,55-63$ and $>63$ years). Third, to explore if associations between RHR and incident T2DM were different in patients with different locations of vascular diseases, analyses were performed per $10 \mathrm{bpm}$ increase in RHR after stratifying patients according to the vascular diagnosis at study inclusion, being CAD, CVD, PAD, or AAA. If inclusion in the SMART study was not due to a recent vascular diagnosis, the first vascular diagnosis in their medical history was used to classify them as CAD, CVD, PAD, or AAA.

All regression analyses were conducted with a crude model (I) and three models to adjust for potential confounding factors. In model II, we adjusted for age and gender and in model III, additional adjustments were carried out for current smoking, SBP, eGFR, the use of medication with possible effect on the RHR and on incident T2DM (beta-blockers, diuretics, calcium channel-blockers, angiotensin-converting enzyme inhibitors, and angiotensin II receptor blockers) and the location of vascular disease at study inclusion (CAD, CVD, PAD, or AAA). The study conclusions are based on this fully adjusted model. Finally, in an exploratory model (IV) we additionally adjusted for variables that may be in the causal pathway between RHR and incident T2DM, being $\mathrm{BMI}$ and fasting glucose. Current smoking, gender, the vascular diagnosis, and the use of medication were included as categorical variables and age, eGFR, SBP, BMI, and fasting glucose were entered as continuous variables.

Analyses were repeated after exclusion of patients with baseline fasting glucose values $\geq 7.0 \mathrm{mmol} / \mathrm{l}$ $(n=212)$ and patients with extreme heart rates $(\leq 50$ $(n=436)$ or $\geq 100 \mathrm{bpm}(n=35))$, to avoid a large effect of patients with impaired fasting glucose or extreme heart rates. Additional exploratory correction for thyroid-stimulating hormone (TSH), another possible factor in the causal pathway between RHR and incident T2DM, was also carried out. Finally, besides correcting for beta-blocker use, analyses were conducted in patients on beta-blocker $(n=1934)$ and not on betablocker $(n=1712)$ treatment separately, as beta-blockers influence both the RHR and the risk for diabetes. SPSS version 15.0.1 was used for all analyses. 


\section{Results}

\section{Baseline characteristics}

Baseline characteristics per quartile of RHR are presented in Table 1. The average RHR increased from $50 \pm 4 \mathrm{bpm}$ in Q1 to $79 \pm 9 \mathrm{bpm}$ in Q4. The mean age of the study population was $59 \pm 10$ years and the percentage of male participants decreased from 81 in Q1 to 66 in Q4. Over the quartiles with increasing RHR, slight increases were observed in fasting serum glucose levels $(\mathrm{Q} 1,5.7 \pm 0.7-\mathrm{Q} 4,5.8 \pm 0.8 \mathrm{mmol} / \mathrm{l})$ and $\mathrm{BMI}$ $\left(\mathrm{Q} 1,26.2 \pm 3.2-\mathrm{Q} 4,26.8 \pm 4.2 \mathrm{~kg} / \mathrm{m}^{2}\right)$.

\section{Follow-up and incident type 2 diabetes}

The total follow-up time in this study was 21535 person-years, with a median follow-up of 5.5 (IQR, 3.2-8.4) years. During this follow-up, 289 patients were diagnosed with incident T2DM and 188 patients died. The unadjusted incidence rate (new cases of T2DM per 1000 person-years of follow-up) increased across quartiles or RHR (from 9.6 (95\% CI, 7.3-12.5) in Q1 to 16.0 (95\% CI, 13.0-19.6) in Q4; Fig. 2A) and with increasing age (from 11.9 (95\% CI, 9.7-14.5) for subjects $<55$ years to 14.2 (95\% CI, 11.6-17.2) for subjects $>63$ years (Fig. 2B)).

\section{RHR and incident T2DM}

Subjects in the highest quartile of RHR (Q4) had a 65\% higher risk of incident T2DM compared with those in the reference Q1 (HR, 1.65; 95\% CI, 1.15-2.36) based on the fully adjusted model (III; Fig. 3). Every 10 bpm increase in RHR was related to a $10 \%$ increase in incident T2DM (HR, 1.10; 95\% CI, 1.00-1.21) in the total population. The point estimates in this paragraph should be interpreted with caution as there is effect modification by age.

\section{Effect modification of age on the relation between RHR and incident T2DM}

Before conducting the analyses, we investigated potential effect modification on a multiplicative scale of the relationship between RHR and incident T2DM for factors that may act as effect modifiers on pathophysiological grounds, by entering cross-products of RHR and the

Table 1 Baseline characteristics according to quartiles of resting heart rate $(n=3646)$. The study population is presented in quartiles according to their resting heart rate.

\begin{tabular}{|c|c|c|c|c|}
\hline & Quartile 1 ( $n=889)$ & Quartile $2(n=965)$ & Quartile $3(n=838)$ & Quartile $4(n=954)$ \\
\hline RHR (mean; bpm) & $50 \pm 4$ & $58 \pm 2$ & $65 \pm 3$ & $79 \pm 9$ \\
\hline RHR (range; bpm) & $31-54$ & $55-61$ & $62-69$ & $70-122$ \\
\hline Male gender $(n ; \%)$ & $723(81)$ & $711(74)$ & $578(69)$ & $628(66)$ \\
\hline Age (years) & $58.9 \pm 9.9$ & $59.0 \pm 10.3$ & $58.2 \pm 10.6$ & $58.7 \pm 10.8$ \\
\hline Current smoking $(n ; \%)$ & $246(28)$ & $289(30)$ & $313(37)$ & $395(41)$ \\
\hline Body mass index $\left(\mathrm{kg} / \mathrm{m}^{2}\right)$ & $26.2 \pm 3.2$ & $26.7 \pm 3.7$ & $26.7 \pm 3.9$ & $26.8 \pm 4.2$ \\
\hline Waist circumference $(\mathrm{cm})$ & $94 \pm 10$ & $95 \pm 11$ & $94 \pm 12$ & $95 \pm 13$ \\
\hline Systolic BP (mmHg) & $138 \pm 22$ & $140 \pm 22$ & $142 \pm 22$ & $14 \overline{4} \pm 21$ \\
\hline Diastolic BP (mmHg) & $80 \pm 11$ & $82 \pm 11$ & $84 \pm 12$ & $85 \pm 12$ \\
\hline \multicolumn{5}{|l|}{ Laboratory parameters } \\
\hline Total cholesterol $(\mathrm{mmol} / \mathrm{l})$ & $4.82 \pm 1.14$ & $4.89 \pm 1.14$ & $5.09 \pm 1.30$ & $5.19 \pm 1.28$ \\
\hline Triglycerides $(\mathrm{mmol} / \mathrm{l})$ & $1.29(0.96-1.80)$ & $1.37(1.00-1.97)$ & $1.43(1.04-2.04)$ & $1.49(1.09-2.14)$ \\
\hline HDL-cholesterol $(\mathrm{mmol} / \mathrm{l})$ & $1.24 \pm 0.36$ & $1.25 \pm 0.37$ & $1.27 \pm 0.40$ & $1.27 \pm 0.40$ \\
\hline LDL-cholesterol (mmol/l) & $2.89 \pm 1.01$ & $2.90 \pm 1.00$ & $3.05 \pm 1.05$ & $3.09 \pm 1.07$ \\
\hline ApoB $(g / l)$ & $0.8 \pm 0.2$ & $0.9 \pm 0.2$ & $0.9 \pm 0.3$ & $0.9 \pm 0.3$ \\
\hline Glucose $(\mathrm{mmol} / \mathrm{l})$ & $5.7 \pm 0.7$ & $5.7 \pm 0.7$ & $5.8 \pm 0.9$ & $5.8 \pm 0.8$ \\
\hline eGFR $\left(\mathrm{ml} / \mathrm{min}\right.$ per $\left.1.73 \mathrm{~m}^{2}\right)$ & $76 \pm 16$ & $76 \pm 16$ & $78 \pm 17$ & $77 \pm 19$ \\
\hline $\operatorname{hsCRP}(\mathrm{mg} / \mathrm{l})$ & $1.5(0.7-3.1)$ & $1.6(0.7-3.2)$ & $1.9(1.0-4.0)$ & $2.5(1.1-4.9)$ \\
\hline $\mathrm{TSH}(\mathrm{mU} / \mathrm{l})$ & $1.70(1.20-2.40)$ & $1.60(1.10-2.30)$ & $1.60(1.10-2.30)$ & $1.70(1.20-2.50)$ \\
\hline \multicolumn{5}{|c|}{ Vascular disease at inclusion $(n ; \%)$} \\
\hline CAD & $605(68)$ & $565(59)$ & $401(48)$ & $344(36)$ \\
\hline CVD & $160(18)$ & $224(23)$ & $239(29)$ & $309(32)$ \\
\hline PAD & $96(11)$ & $131(14)$ & $144(17)$ & $226(24)$ \\
\hline AAA & $28(3)$ & $45(5)$ & $54(6)$ & $75(8)$ \\
\hline \multicolumn{5}{|l|}{ Medication use $(n ; \%)$} \\
\hline Platelet aggregation inhib & $721(81)$ & $769(80)$ & $616(74)$ & $624(65)$ \\
\hline Oral anticoagulation & $58(7)$ & $79(8)$ & $74(9)$ & $106(11)$ \\
\hline Lipid-lowering med & $596(67)$ & $639(66)$ & $519(62)$ & $559(59)$ \\
\hline BP-lowering med & $705(79)$ & $722(75)$ & $578(69)$ & $611(64)$ \\
\hline Beta-blocker & $604(68)$ & $580(60)$ & $403(48)$ & $347(36)$ \\
\hline Diuretic & $92(10)$ & $154(16)$ & $135(16)$ & $195(20)$ \\
\hline ACE-i/ARB & $251(28)$ & $294(31)$ & $271(32)$ & $336(35)$ \\
\hline Calcium channel blocker & $188(21)$ & $204(21)$ & 157 (19) & $166(17)$ \\
\hline
\end{tabular}

$\mathrm{BP}$, blood pressure; bpm, beats per minute; ApoB, apolipoprotein B; eGFR, estimated glomerular filtration rate; hsCRP, high-sensitivity C-reactive protein; TSH, thyroid-stimulating hormone; inhib, inhibitors; med, medication; ACE-i, angiotensin-converting enzyme inhibitor; ARB, angiotensin receptor blocker. 
A According to quartiles of resting heart rate
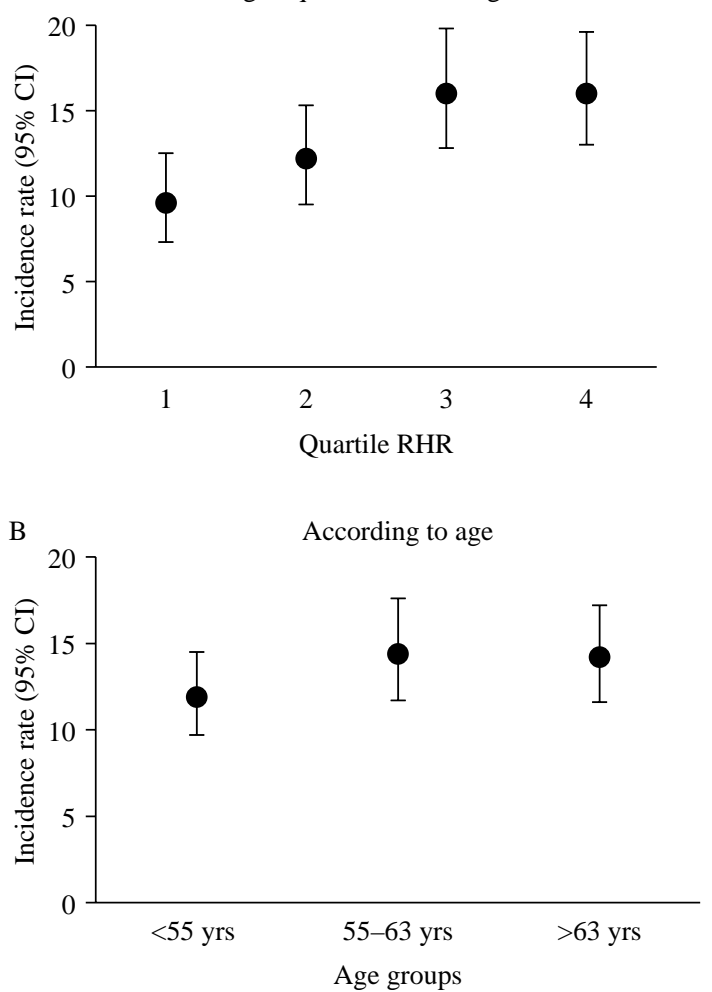

Figure 2 Incidence rates for T2DM in patients with clinically manifest vascular diseases. Incidence rates are expressed as cases per 1000 person-years of study follow-up.

possible effect modifier in the Cox proportional hazard model. Gender was not a significant effect modifier $(P=0.38)$, nor was beta-blocker use $(P=0.16)$. Only age appeared to modify the relation between RHR and incident T2DM significantly ( $P$ for interaction $=0.048$ ). Stratification for age in three groups (tertiles) showed that in subjects aged between 55 and 63 years, every 10 bpm increase in RHR increased the risk of incident T2DM by $22 \%$ (HR, $1.22 ; 95 \% \mathrm{CI}, 1.04-1.43)$, while there was no increased risk of T2DM with increasing RHR in patients aged <55 years (HR, 1.08; 95\% CI, 0.91-1.30) and patients aged > 73 years (HR, 1.00; 95\% CI, 0.84-1.18; Table 2). Further exploratory analyses in smaller age groups (quintiles) confirmed the finding that the relation between increased RHR and incident T2DM is absent in the youngest and oldest groups of patients, and is present in middle-aged patients (between 51 and 68 years in this analysis).

\section{Exploratory analyses}

In an exploratory analysis (model IV), the relation between RHR and incident T2DM was attenuated to a large extent by adding BMI and fasting serum glucose to the fully adjusted model, indicating that BMI and serum glucose levels are part of the causal pathway between sympathetic nerve activity measured with RHR and incident T2DM (Table 2).

Furthermore, in separate analyses after exclusion of patients with a baseline fasting plasma glucose level $\geq 7.0 \mathrm{mmol} / \mathrm{l}$ or after exclusion of patients with an RHR $\leq 50$ or $\geq 100 \mathrm{bpm}$, the results were not essentially different. Adjustment for levels of TSH did not change the results either. Each $10 \mathrm{bpm}$ increase in RHR was associated with a 19\% higher risk for incident T2DM in patients on beta-blocker treatment (HR, 1.19; 95\% CI, 1.04-1.37) and only with a 3\% higher risk in patients without beta-blocker use (HR, 1.03; 95\% CI, 0.90-1.18); however, this difference was not statistically significant $(P$ for interaction $=0.16)$.

\section{RHR and incident T2DM according to location of vascular disease}

The relation between RHR and incident T2DM was not significantly different between patients with different locations of vascular disease $(P$ for interaction $=0.72$ for $\mathrm{CAD}, 0.69$ for $\mathrm{CVD}, 0.82$ for $\mathrm{PAD}$, and 0.84 for AAA); every $10 \mathrm{bpm}$ increase in RHR was associated with a 13\% higher risk for T2DM in patients with CVD and PAD (HR, 1.13; 95\% CI, 0.93-1.37 and HR, 1.13; 95\% CI, 0.93-1.37 respectively), an 8\% higher risk in patients with CAD (HR, 1.08; 95\% CI, 0.92-1.26), and a 5\% higher risk in patients with AAA (HR, 1.05; 95\% CI, 0.78-1.41; Fig. 4).

\section{Discussion}

In this study it is shown that an increased RHR is associated with an increased risk for developing T2DM in patients with clinically manifest vascular diseases,

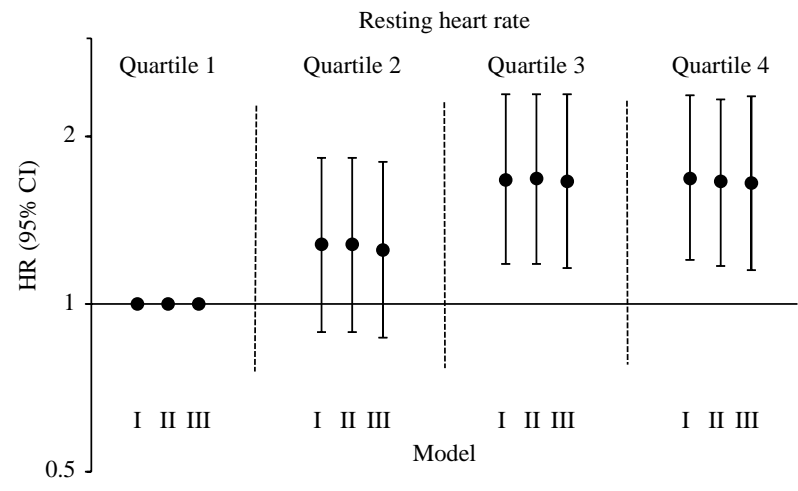

Figure 3 The risk of increasing RHR on incident T2DM in patients with clinically manifest vascular diseases. Results are expressed as hazard ratios $(\mathrm{HR})$ with $95 \% \mathrm{Cl}$ per quartile of $\mathrm{RHR}$, relative to quartile 1 (reference). Model I: crude; model II: age and gender; model III: age, gender, beta-blocker use, diuretic use, calciumblocker use, use of angiotensin-converting enzyme inhibitor or angiotensin receptor blocker, current smoking, estimated glomerular filtration rate, systolic blood pressure, location of vascular disease (CAD, CVD, PAD or AAA). 
Table 2 The risk of RHR per 10 bpm on incident T2DM in patients with clinically manifest vascular diseases, after stratification in three age groups. Results are expressed as hazard ratios (HR) with $95 \% \mathrm{Cl}$ per $10 \mathrm{bpm}$ increase in RHR.

\begin{tabular}{lccc}
\hline & \multicolumn{3}{c}{ Age groups } \\
\cline { 2 - 4 } & 1 & 2 & 3 \\
\hline$n$ & 1255 & 1127 & 1264 \\
Age (median; range) & $49(19-54)$ & $59(55-63)$ & $69(64-80)$ \\
RHR (median; range) & $63 \pm 12(31-117)$ & $63 \pm 12(39-114)$ & $63 \pm 13(36-122)$ \\
No. of events & 94 & 94 & 101 \\
Model HR (95\% CI) & $1.13(0.95-1.33)$ & $1.32(1.14-1.53)$ & $0.94(0.80-1.10)$ \\
I & $1.13(0.96-1.34)$ & $1.31(1.13-1.51)$ & $0.92(0.79-1.08)$ \\
II & $1.08(0.91-1.30)$ & $1.22(1.04-1.43)$ & $1.00(0.84-1.18)$ \\
III & $1.01(0.83-1.23)$ & $1.03(0.86-1.22)$ & $0.92(0.76-1.10)$ \\
IV &
\end{tabular}

Model I: crude; model II: age and gender; model III: age, gender, beta-blocker use, diuretic use, calcium blocker use, use of angiotensin-converting enzyme inhibitor or angiotensin receptor blocker, current smoking, estimated glomerular filtration rate, systolic blood pressure, and inclusion diagnosis (CAD, CVD, PAD or AAA); model IV (exploratory): model III and body mass index and fasting glucose.

particularly in middle-aged (55-63 years) patients, independently of the location of vascular disease or beta-blocker use. These results are in accordance with previous findings in healthy subjects and in subjects with increased BMI or impaired glucose tolerance $(15,16,17,18,19,20)$. As patients with clinically manifest vascular diseases have an increased sympathetic nerve activity (26), reflected by an increased RHR, increased sympathetic tone can be considered to be part of shared pathophysiological pathways for developing diabetes and atherosclerotic vascular diseases, next to insulin resistance and low-grade inflammation as proposed in the 'common soil' hypothesis (25).

The overall 10\% risk of developing T2DM (HR, 1.10; 95\% CI, 1.00-1.21) for every $10 \mathrm{bpm}$ increase in RHR in the present study fits within the range of risks in other study populations $(15,16,17,18,19,20)$. The reported risks in various populations are remarkably similar although population characteristics vary widely. Also, the method for recording RHR varied across studies including ECG, heart rate meter, or pulse palpation.

In this study it is shown that age is an important effect modifier in the relation between RHR and incident T2DM. The risk for incident T2DM is particularly high in middle-aged patients (age range 55-63 years), while there is no clear relation in younger and older patients. Main analyses were performed in tertiles of age. This was an arbitrary decision balancing statistical power and contrast between patient groups. The results were confirmed in exploratory analyses in smaller age groups (quintiles). In the other studies there was no statistically significant interaction of age on the relation between RHR and incident diabetes $(15,16,18)$ or at least this was not reported $(17,19,20)$. In a study conducted in healthy Chinese women, stratified analyses for age revealed that the effect of RHR was larger in women $<55$ years of age compared with women $>55$ years of age, although this was not a statistically significant difference (18). Furthermore, the study conducted in the youngest population (mean age $44 \pm 7$ years) reports the highest risks of developing diabetes per s.D. increase in RHR: HR 1.37 (95\% CI, 1.29-1.45) for men and HR 1.46 (95\% CI, 1.31-1.62) for women (19) compared with the HR ranging from 1.10 to 1.27 in the other populations (mean age ranging from 48 to 54 years) $(15,16,18,20)$. Although drawing a firm conclusion on the basis of these data is not appropriate, an elevated RHR seems to be associated with a higher risk of incident T2DM in younger subjects or subjects at middle age than in older subjects.

Different pathophysiological explanations for the relation between RHR and incident T2DM may be considered. RHR is an indicator of sympathetic activity (12) and an increased sympathetic nerve system induces both acute and chronic insulin resistance $(9,10,11)$. The major organs involved in insulin

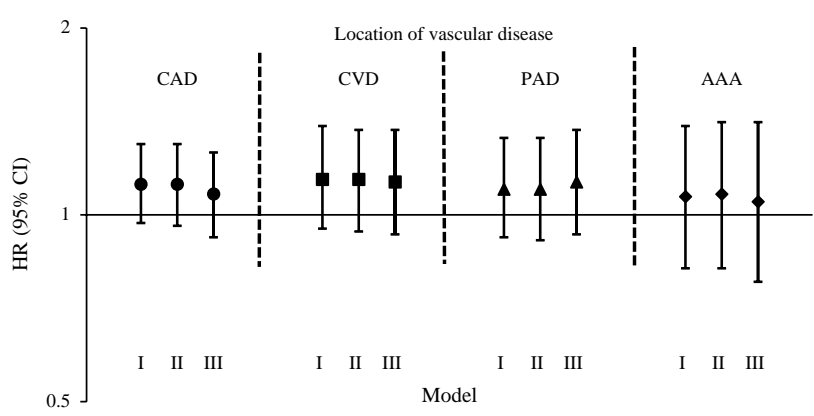

Figure 4 The risk of RHR per $10 \mathrm{bpm}$ on incident T2DM in patients with clinically manifest vascular diseases, stratified for the location of vascular disease. Results are expressed as hazard ratios (HR) with $95 \% \mathrm{Cl}$ per $10 \mathrm{bpm}$ increase in RHR for patients with different locations of vascular diseases. Model I: crude; model II: age and gender; model III: age, gender, beta-blocker use, diuretic use, calcium-blocker use, use of angiotensin-converting enzyme inhibitor or angiotensin receptor blocker, current smoking, estimated glomerular filtration rate, systolic blood pressure, location of vascular disease (CAD, CVD, PAD, or AAA). 
secretion, glucose production and glucose metabolism, including the pancreas, liver and skeletal muscle, are innervated by autonomic nerves $(28,29,30)$. Stimulation of $\beta$-adrenergic receptors causes a reduction in the insulin-stimulated uptake of glucose (31), an increase in the proportion of insulin-resistant fasttwitch muscle fibers in rats (32), and most importantly, vasoconstriction leading to a decreased skeletal muscle blood flow, with impaired glucose uptake into the muscle cells as a result (33).

Both insulin resistance and autonomic dysfunction are the result of, and can be influenced by, physical inactivity and obesity, which are established risk factors for the development of T2DM $(34,35)$, and are also known to influence autonomic function $(36,37)$. Obesity and fasting serum glucose levels are very likely to be part of the causal pathway between RHR and incident T2DM, as shown in the present study by attenuation of the results after adding BMI and fasting serum glucose levels to the regression model. However, obesity and elevated fasting glucose levels could have been the result of autonomic dysfunction (elevated RHR) during the patients' lives, or could have been caused by the high RHR. Obesity is a cause of insulin resistance, which in turn leads to increased sympathetic nerve activity, and to increased glucose levels progressing to overt T2DM. However, cause and effect could also be the other way around. Increased sympathetic nerve activity, as measured by RHR, could also lead to higher BMI and elevated plasma glucose and insulin levels with progression to T2DM. Which of these two explanations is true cannot be concluded from our study. As discussed before, sympathetic nerve activation can be both the cause and the consequence of insulin resistance. The recent finding that reducing sympathetic nerve activity, by renal sympathetic denervation (38), causes improvements in glucose metabolism and insulin sensitivity further underlines the pathophysiological role of the sympathetic nerve system in the development of insulin resistance.

The overall association between RHR and incident T2DM in the present study is found despite a high prevalence of beta-blocker use (53\%). It seems that the predictive role of RHR in patients on beta-blocker treatment is greater (HR, 1.19; 95\% CI, 1.04-1.37) than those without beta-blocker use (HR, 1.03 95\% CI, 0.90-1.18), although this was not statistically significant. This is not surprising considering the fact that beta-blockers favor insulin resistance and increase the risk of developing diabetes (39).

The results of the present study are in line with previous results obtained in the general population $(15,16,17,18,19,20)$, but are of potentially greater clinical significance because of the possible therapeutic implications. RHR is a noninvasive, nonexpensive, and easily obtainable parameter, and therefore easily applicable in clinical practice, in contrast to other measurements that reflect sympathetic nerve activity such as MSNA or plasma noradrenaline levels. Prevention of diabetes in patients with vascular diseases is important, as patients with diabetes have an increased risk for (cardiovascular) morbidity and mortality $(21,22)$ and a reduced quality of life (23). Reducing the risk of T2DM is feasible with lifestyle interventions, such as increasing physical activity and reducing body weight, and with medical treatment $(24,40)$.

Strengths of this study include the prospective cohort design and the large sample size of a clinically relevant and well-characterized group of patients with various locations of clinically manifest vascular diseases. Furthermore, RHR was accurately and consistently measured with ECG and the incidence of T2DM was thoroughly assessed. Assessment of several clinical and laboratory parameters allowed for identification of possible confounding factors.

Study limitations need to be considered. As we excluded subjects who died or were lost to follow-up before the assessment of diabetes started in 2006, and diabetes is associated with a higher mortality, this could have affected the results of this study toward the null hypothesis. This effect is possibly of greatest influence in the older age group, as older persons are at the highest risk of death, which could be an explanation for the absence of a relation between RHR and incident diabetes in this group. Unfortunately, serum insulin levels were not routinely measured in our study, so we could not adjust our exploratory model for insulin resistance expressed as homeostasis model assessment of insulin resistance (HOMA). However, by adjusting for BMI and fasting serum glucose, the role of insulin resistance can be explored as both parameters are strong determinants of HOMA. Finally, physical activity and cardiorespiratory fitness, factors in the causal pathway of RHR and incident T2DM, were not assessed in this study. In the exploratory analyses, we adjusted for other factors in the causal pathway between RHR and incident diabetes (BMI and fasting serum glucose levels). As not only BMI and fasting serum glucose levels but also variables such as age, gender, and smoking are closely related to physical activity and cardiorespiratory fitness, we believe the residual confounding effect in the exploratory analysis of physical activity and cardiorespiratory fitness is limited.

In conclusion, increased RHR, an indicator of increased sympathetic nerve activity, is associated with an increased risk for incident T2DM in patients with clinically manifest vascular diseases. This risk was particularly elevated in middle-aged patients and was irrespective of the location of vascular disease and of the use of beta-blockers.

\section{Declaration of interest}

The authors declare that there is no conflict of interest that could be perceived as prejudicing the impartiality of the research reported. 


\section{Funding}

The SMART study is financially supported by the University Medical Center Utrecht. The funding sources had no role in the collection, analysis or interpretation of the data, or in the decision to submit the paper for publication.

\section{Acknowledgements}

We gratefully acknowledge the contribution of the SMART research nurses, R van Petersen (data manager), A G Pijl (vascular manager) and the participants of the SMART Study Group members: A Algra MD, PhD; Y van der Graaf, MD, PhD; D E Grobbee, MD, PhD; G E H M Rutten, MD, PhD, Julius Center for Health Sciences and Primary care; F L J Visseren, MD, PhD, Department of Vascular Medicine; F L Moll, MD, PhD, Department of Vascular Surgery; L J Kappelle, MD, PhD, Department of Neurology; W P T M Mali, MD PhD, Department of Radiology; PA Doevendans, MD, PhD, Department of Cardiology, all in the University Medical Center Utrecht, Utrecht, The Netherlands.

\section{References}

1 Grassi G, Dell'Oro R, Facchini A, Quarti TF, Bolla GB \& Mancia G. Effect of central and peripheral body fat distribution on sympathetic and baroreflex function in obese normotensives. Journal of Hypertension 200422 2363-2369. (doi:10.1097/ 00004872-200412000-00019)

2 Esteghamati A, Khalizadeh O, Anvari M, Rashidi A, Mokhtari M \& Nakhjavani M. Association of serum leptin levels with homeostasis model assessment-estimated insulin resistance and metabolic syndrome: the key role of central obesity. Metabolic Syndrome and Related Disorders 20097 447-452. (doi:10.1089/met.2008. 0100)

3 Anderson EA, Hoffman RP, Balon TW, Sinkey CA \& Mark AL. Hyperinsulinemia produces both sympathetic neural activation and vasodilation in normal humans. Journal of Clinical Investigation 199187 2246-2252. (doi:10.1172/JCI115260)

4 Sauter A, Goldstein M, Engel J \& Ueta K. Effect of insulin on central catecholamines. Brain Research 1983260 330-333. (doi:10. 1016/0006-8993(83)90691-1)

5 Ward KR, Bardgett JF, Wolfgang L \& Stocker SD. Sympathetic response to insulin is mediated by melanocortin $3 / 4$ receptors in the hypothalamic paraventricular nucleus. Hypertension 201157 435-441. (doi:10.1161/HYPERTENSIONAHA.110.160671)

6 Narkiewicz K, Kato M, Phillips BG, Pesek CA, Choe I, Winnicki M, Palatini P, Sivitz WI \& Somers VK. Leptin interacts with heart rate but not sympathetic nerve traffic in healthy male subjects. Journal of Hypertension 200119 1089-1094. (doi:10.1097/ 00004872-200106000-00014)

7 Tartaglia LA, Dembski M, Weng X, Deng N, Culpepper J, Devos R, Richards GJ, Campfield LA, Clark FT, Deeds J, Muir C, Sanker S, Moriarty A, Moore KJ, Smutko JS, Mays GG, Wool EA, Monroe CA \& Tepper RI. Identification and expression cloning of a leptin receptor, OB-R. Cell 199583 1263-1271. (doi:10.1016/00928674(95)90151-5)

8 Shek EW, Brands MW \& Hall JE. Chronic leptin infusion increases arterial pressure. Hypertension 199831 409-414.

9 Jamerson KA, Julius S, Gudbrandsson T, Andersson O \& Brant DO. Reflex sympathetic activation induces acute insulin resistance in the human forearm. Hypertension 199321 618-623.

10 Masuo K, Mikami H, Ogihara T \& Tuck ML. Sympathetic nerve hyperactivity precedes hyperinsulinemia and blood pressure elevation in a young, nonobese Japanese population. American Journal of Hypertension 199710 77-83. (doi:10.1016/S08957061(96)00303-2)

11 Palatini P, Mos L, Santonastaso M, Zanatta N, Mormino P, Saladini F, Bortolazzi A, Cozzio S \& Garavelli G. Resting heart rate as a predictor of body weight gain in the early stage of hypertension. Obesity 2011 19 618-623. (doi:10.1038/oby.2010.191)
12 Grassi G, Vailati S, Bertinieri G, Seravalle G, Stella ML, Dell'Oro R \& Mancia G. Heart rate as marker of sympathetic activity. Journal of Hypertension 199816 1635-1639. (doi:10.1097/00004872199816110-00010)

13 Ho JE, Bittner V, Demicco DA, Breazna A, Deedwania PC \& Waters DD. Usefulness of heart rate at rest as a predictor of mortality, hospitalization for heart failure, myocardial infarction, and stroke in patients with stable coronary heart disease (data from the Treating to New Targets [TNT] trial). American Journal of Cardiology 2010105 905-911. (doi:10.1016/j.amjcard.2009. 11.035)

14 Palatini P \& Julius S. Elevated heart rate: a major risk factor for cardiovascular disease. Clinical and Experimental Hypertension 200426 637-644. (doi:10.1081/CEH-200031959)

15 Carnethon MR, Golden SH, Folsom AR, Haskell W \& Liao D. Prospective investigation of autonomic nervous system function and the development of type 2 diabetes: the Atherosclerosis Risk in Communities study, 1987-1998. Circulation $2003 \mathbf{1 0 7}$ 2190-2195. (doi:10.1161/01.CIR.0000066324.74807.95)

16 Carnethon MR, Yan L, Greenland P, Garside DB, Dyer AR, Metzger B \& Daviglus ML. Resting heart rate in middle age and diabetes development in older age. Diabetes Care 200831 335-339. (doi:10.2337/dc07-0874)

17 Shigetoh Y, Adachi H, Yamagishi S, Enomoto M, Fukami A, Otsuka M, Kumagae S, Furuki K, Nanjo Y \& Imaizumi T. Higher heart rate may predispose to obesity and diabetes mellitus: 20-year prospective study in a general population. American Journal of Hypertension 200922 151-155. (doi:10.1038/ajh.2008.331)

18 Zhang X, Shu XO, Xiang YB, Yang G, Li H, Cai H, Gao YT \& Zheng W. Resting heart rate and risk of type 2 diabetes in women. International Journal of Epidemiology 201039 900-906. (doi:10. 1093/ije/dyq068)

19 Nagaya T, Yoshida H, Takahashi H \& Kawai M. Resting heart rate and blood pressure, independent of each other, proportionally raise the risk for type-2 diabetes mellitus. International Journal of Epidemiology 201039 215-222. (doi:10. 1093/ije/dyp229)

20 Carnethon MR, Prineas RJ, Temprosa M, Zhang ZM, Uwaifo G \& Molitch ME. The association among autonomic nervous system function, incident diabetes, and intervention arm in the Diabetes Prevention Program. Diabetes Care 200629 914-919. (doi:10. 2337/diacare.29.04.06.dc05-1729)

21 Eberly LE, Cohen JD, Prineas R \& Yang L. Impact of incident diabetes and incident nonfatal cardiovascular disease on 18-year mortality: the multiple risk factor intervention trial experience. Diabetes Care 200326 848-854. (doi:10.2337/diacare.26.3.848)

22 Kannel WB \& McGee DL. Diabetes and glucose tolerance as risk factors for cardiovascular disease: the Framingham study. Diabetes Care 19792 120-126. (doi:10.2337/diacare.2.2.120)

23 Gough SC, Kragh N, Ploug UJ \& Hammer M. Impact of obesity and type 2 diabetes on health-related quality of life in the general population in England. Diabetes, Metabolic Syndrome and Obesity: Targets and Therapy 20092 179-184. (doi:10.2147/DMSOTT. S7088)

24 Knowler WC, Barrett-Connor E, Fowler SE, Hamman RF, Lachin JM, Walker EA \& Nathan DM. Reduction in the incidence of type 2 diabetes with lifestyle intervention or metformin. New England Journal of Medicine 2002346 393-403. (doi:10.1056/ NEJMoa012512)

25 Stern MP. Diabetes and cardiovascular disease. The "common soil" hypothesis. Diabetes $1995 \mathbf{4 4}$ 369-374. (doi:10.2337/diabetes. 44.4.369)

26 Grassi G, Arenare F, Pieruzzi F, Brambilla G \& Mancia G. Sympathetic activation in cardiovascular and renal disease. Journal of Nephrology 200922 190-195.

27 Simons PC, Algra A, van de Laak MF, Grobbee DE \& van der Graaf Y. Second manifestations of arterial disease (SMART) study: rationale and design. European Journal of Epidemiology 199915 773-781. (doi:10.1023/A:1007621514757) 
28 Reaven GM, Lithell H \& Landsberg L. Hypertension and associated metabolic abnormalities - the role of insulin resistance and the sympathoadrenal system. New England Journal of Medicine 1996 334 374-381. (doi:10.1056/NEJM199602083340607)

29 Shimazu T. Innervation of the liver and glucoregulation: roles of the hypothalamus and autonomic nerves. Nutrition 199612 65-66.

30 Nonogaki K. New insights into sympathetic regulation of glucose and fat metabolism. Diabetologia 200043 533-549. (doi:10. 1007/s001250051341)

31 Deibert DC \& DeFronzo RA. Epinephrine-induced insulin resistance in man. Journal of Clinical Investigation 198065 717-721. (doi:10.1172/JCI109718)

32 Zeman RJ, Ludemann R, Easton TG \& Etlinger JD. Slow to fast alterations in skeletal muscle fibers caused by clenbuterol, a beta 2-receptor agonist. American Journal of Physiology $1988 \mathbf{2 5 4}$ E726-E732.

33 Julius S, Gudbrandsson T, Jamerson K \& Andersson O. The interconnection between sympathetics, microcirculation, and insulin resistance in hypertension. Blood Pressure 19921 9-19. (doi:10.3109/08037059209065119)

34 Ivy JL. Role of exercise training in the prevention and treatment of insulin resistance and non-insulin-dependent diabetes mellitus. Sports Medicine 199724 321-336. (doi:10.2165/00007256199724050-00004)

35 Vazquez G, Duval S, Jacobs DR Jr \& Silventoinen K. Comparison of body mass index, waist circumference, and waist/hip ratio in predicting incident diabetes: a meta-analysis. Epidemiologic Reviews 200729 115-128. (doi:10.1093/epirev/mxm008)
36 Blomqvist CG \& Saltin B. Cardiovascular adaptations to physical training. Annual Review of Physiology $1983 \mathbf{4 5} 169-189$. (doi:10. 1146/annurev.ph.45.030183.001125)

37 Esler M, Straznicky N, Eikelis N, Masuo K, Lambert G \& Lambert E. Mechanisms of sympathetic activation in obesity-related hypertension. Hypertension $2006 \mathbf{4 8} 787-796$. (doi:10.1161/01.HYP. $0000242642.42177 .49)$

38 Mahfoud F, Schlaich M, Kindermann I, Ukena C, Cremers B, Brandt MC, Hoppe UC, Vonend O, Rump LC, Sobotka PA, Krum H, Esler M \& Bohm M. Effect of renal sympathetic denervation on glucose metabolism in patients with resistant hypertension: a pilot study. Circulation 2011123 1940-1946. (doi:10.1161/CIRCULATIONAHA.110.991869)

39 Vardeny O, Uno H, Braunwald E, Rouleau JL, Gersh B, Maggioni AP, Domanski M, Pfeffer MA \& Solomon SD. Opposing effects of $\beta$ blockers and angiotensin-converting enzyme inhibitors on development of new-onset diabetes mellitus in patients with stable coronary artery disease. American Journal of Cardiology 2011 107 1705-1709. (doi:10.1016/j.amjcard.2011.01.064)

40 Zinman B, Harris SB, Neuman J, Gerstein HC, Retnakaran RR, Raboud J, Qi Y \& Hanley AJ. Low-dose combination therapy with rosiglitazone and metformin to prevent type 2 diabetes mellitus (CANOE trial): a double-blind randomised controlled study. Lancet 2010376 103-111. (doi:10.1016/S0140-6736(10)60746-5)

Received 22 November 2011

Revised version received 10 January 2012

Accepted 25 January 2012 